\title{
Shadowing in the nuclear photoabsorption above the resonance region
}

\author{
N. Bianchi, E. De Sanctis, M. Mirazita, V. Muccifora \\ Istituto Nazionale di Fisica Nucleare - Laboratori Nazionali di Frascati, \\ C.P. 13, I-00044 Frascati, Italy
}

February 26, 2018

\begin{abstract}
A model based on the hadronic fluctuations of the real photon is developed to describe the total photonucleon and photonuclear cross sections in the energy region above the nucleon resonances. The hadronic spectral function of the photon is derived including the finite width of vector-meson resonances and the quark-antiquark continuum. The shadowing effect is evaluated considering the effective interaction of the hadronic component with the bound nucleons within a Glauber-Gribov multiple scattering theory. The low energy onset of the shadowing effect is interpreted as a possible signature of a modification of the hadronic spectral function in the nuclear medium. A decrease of the $\rho$-meson mass in nuclei is suggested for a better explanation of the experimental data.
\end{abstract}

PACS n. 25.20.Gf, 12.40.Vv

Keywords: photoabsorption, shadowing, nuclear medium effect 


\section{Introduction}

The reduction of the absorption strength of high energy real photons on nuclei is known as shadowing effect. This effect is generally described considering the real photon as a superposition of a bare photon and of a hadronic fluctuation with the same quantum numbers $\left(J^{P C}=1^{--}\right)$. Within this model the shadowing is produced by the coherent multiple scattering of the hadronic intermediate state on different nucleons inside the nucleus. The amount of the shadowing mainly depends on macroscopic nuclear parameters like the mass number $\mathrm{A}$ and the radius $r_{A}$, and on properties of the hadronic fluctuation like the coherence length $\lambda_{h}$ and the interaction cross section $\sigma_{h N}$ with the nucleon.

In earliest simple models [1], the hadronic component of the photon is given by the low-lying vector mesons $\rho, \omega$ and $\phi$. These Vector Meson Dominance (VMD) models qualitatively reproduce the photonuclear absorption cross section behavior in the several $\mathrm{GeV}$ domain [2]. Generalized Vector Meson Dominance (GVMD) models 3], which include higher mass vector mesons and non diagonal terms, better explain higher energy real photon absorption and virtual photon absorption in deep inelastic electron scattering.

On the contrary, at low real photon energies most of the calculations fail to reproduce the experimental results [4]. Two recent VMD calculations, that describe the vectormeson mass distributions with $\delta$-functions $[5$, 6 ] and consider an energy independent vector-meson nucleon cross section $\sigma_{V N}$ [5], do not predict the nuclear damping of the photoabsorption strength observed below $2 \mathrm{GeV}$, as shown in Fig. 1 for the carbon case. In addition they also underestimate the experimental shadowing effect between 2 and $3 \mathrm{GeV}$. The result of a GVMD calculation [7], in which the energy behavior of $\sigma_{V N}$ cross section is taken into account, is also given in Fig. 1. It clearly shows a better agreement with the experimental shadowing ratio, but it is not able to reproduce the absolute value of the total photonuclear cross section.

The shadowing phenomena, also observed in deep inelastic lepton nucleus scattering, is also studied within a VMD model in which the photon hadronic spectral function is derived from the empirical cross sections of the $e^{+} e^{-} \rightarrow$ hadrons processes [8]. Besides the vector meson mass spectra, this model also includes the low en$\operatorname{ergy} \pi^{+} \pi^{-}$non-resonant production, and the high energy quark-antiquark continuum.

The importance of the hadronic spectral function in the description of the process is shown in Fig. 2 where the coherence length $\lambda_{V}=2 k / m_{V}^{2}$ of vector mesons of lower mass $m_{V}$ are given as a function of the photon energy $k$. The shadowing effect starts to manifest at an energy for which $\lambda_{V}$ is bigger than the typical intranucleon distance $\left(d_{N N} \sim 1.8 \mathrm{fm}\right)$ so that scattering on at least two nucleons is possible. Then the strength of the effect starts to saturate at an energy for which $\lambda_{V}$ is bigger than the nu-

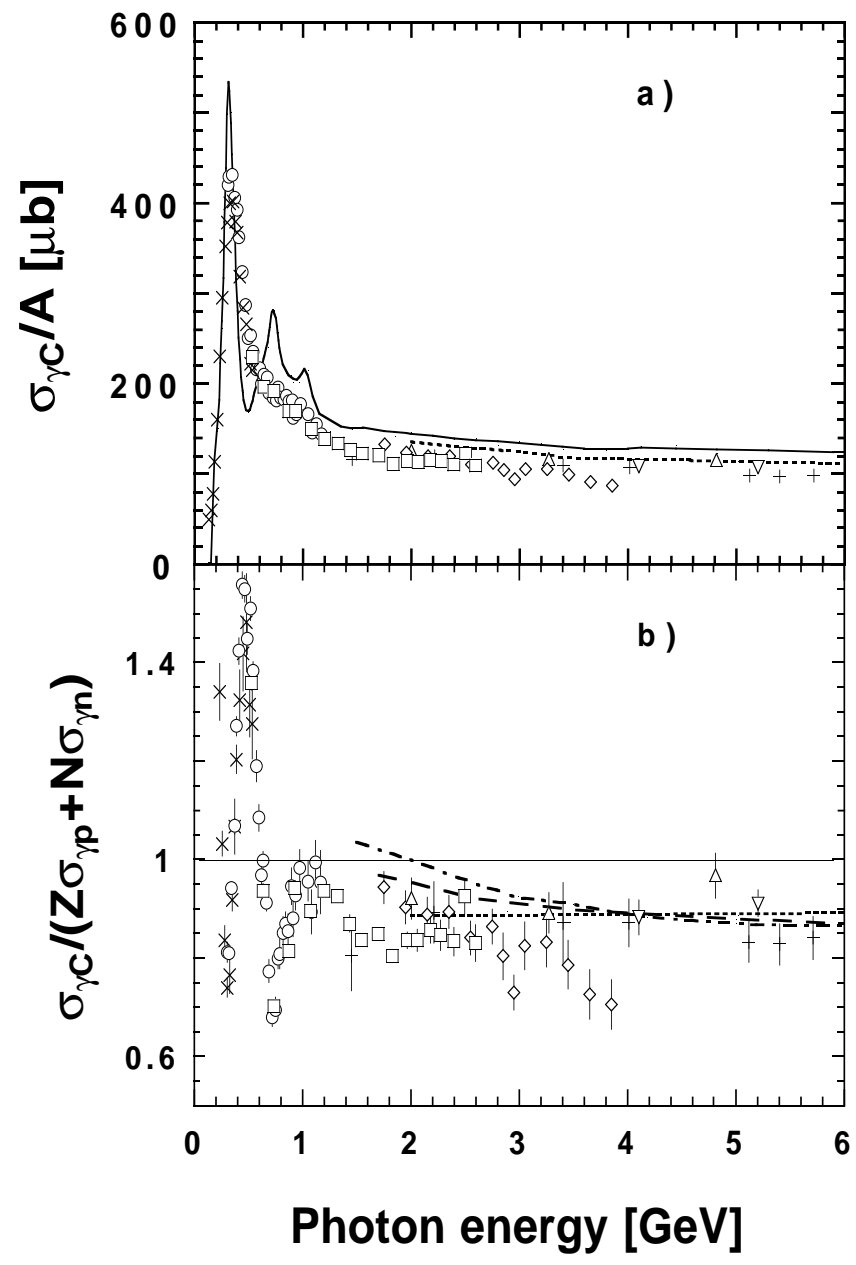

Figure 1: a) Total photonuclear cross section and b) ratio to the photonucleon cross section for carbon. Different symbols refer to different experiments. Also shown in a) is the total cross section on hydrogen (thin solid line). Dashed [5], dot-dashed [6] and dotted [7] lines are two VMD and one GVMD predictions.

clear size $\left(\sim 2 r_{A}\right)$. Clearly a low energy shadowing can be only induced by the lowest mass hadronic components of the photon spectral function and by their possible modifications in the nuclear medium. Both the reduction of the vector-meson mass [9, 10] and the modification of the $\rho$-meson spectral function [11, 12] can decrease the photon energy at which the coherence length starts to exceed the intranucleon distance thus producing an earlier onset of the shadowing effect.

In this paper a model is derived to describe the photonucleon and the photonuclear total absorption cross sections above the nucleon resonance region $(k \geq 1.65 \mathrm{GeV})$. In particular the experimental hadronic spectral function, vector-meson nucleon cross sections and effective $\rho$-coupling constant are taken into account. A possible modification of the hadronic spectral function inside the nuclear medium is also considered. 


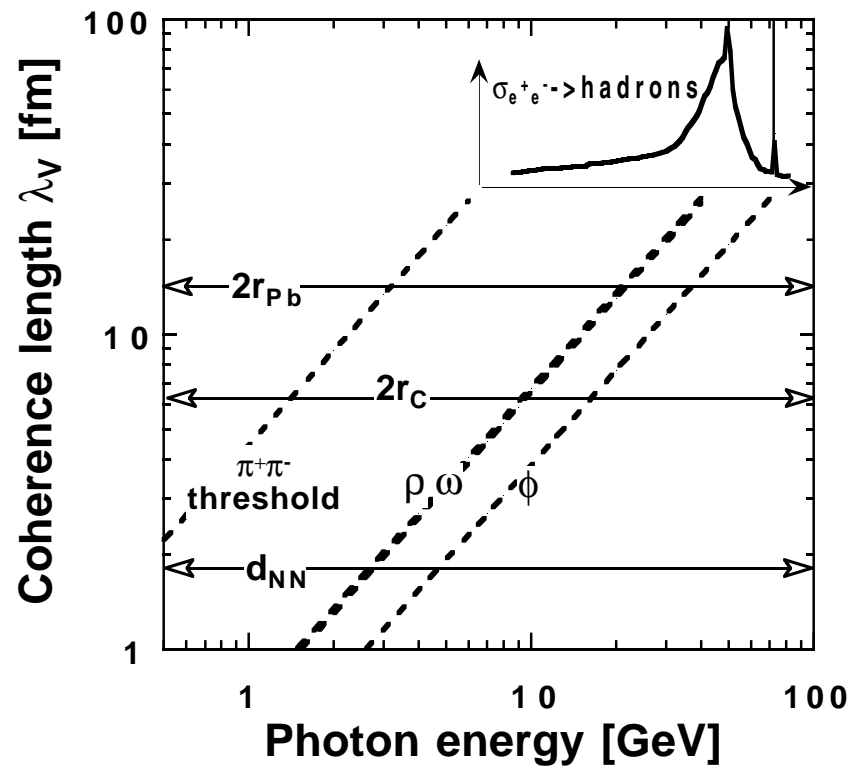

Figure 2: Coherence length of the hadronic spectral function as a function of the photon energy. The average intranucleon distance $d_{N N}$, the carbon $2 r_{C}$ and lead $2 r_{P b}$ nuclear diameters are also shown.

\section{Model}

In the description of the photohadronic absorption process, the physical photon is considered as a superposition of a bare photon and a hadronic component made up of a quark-antiquark state $(q \bar{q})$. The photonucleon cross section $\sigma_{\gamma N}$ is decomposed in a term $\sigma_{\gamma N}^{d i r}$ due to the direct coupling of the bare photon with the nucleon and an hadronic term $\sigma_{\gamma N}^{h a d}$.

At small total center of mass energy the hadronic components of the absorbed photon are mainly formed by strongly correlated $q \bar{q}$ pairs, while at higher energy $q \bar{q}$ pairs from the so-called continuum are also important.

\subsection{Photoabsorption on the nucleon}

The hadronic contribution to the photoabsorption cross section on the proton is expressed by a spectral relation of the form [5, 7]:

$$
\sigma_{\gamma p}^{h a d}(k)=4 \pi \alpha_{e m} \int_{s_{0}}^{s_{u}} \frac{d \mu^{2}}{\mu^{2}} \Pi\left(\mu^{2}\right) \sigma_{h p}\left(\mu^{2}, k\right),
$$

being $\Pi\left(\mu^{2}\right)$ the spectrum of the hadronic fluctuation of mass $\mu$ and $\sigma_{h p}$ the effective hadron-proton cross section. The integration limits are the two pion production threshold $s_{0} \equiv\left(2 m_{\pi}\right)^{2}$ and $s_{u}=\left(\sqrt{s}-m_{p}\right)^{2}$ with $s$ the total center of mass energy and $m_{p}$ the proton mass. The hadronic spectral function of the photon $\Pi\left(\mu^{2}\right)$ is related to the measured cross section of the $e^{+} e^{-} \rightarrow$ hadrons process by

$$
\Pi(s)=\frac{1}{12 \pi^{2}} \frac{\sigma_{e^{+} e^{-} \rightarrow \text { hadrons }}(s)}{\sigma_{e^{+} e^{-} \rightarrow \mu^{+} \mu^{-}}(s)} .
$$

At low energy $\left(s \leq s_{1}=m_{\phi}^{2} \approx 1 \mathrm{GeV}^{2}\right), \Pi(s)$ is dominated by the resonance contribution $\Pi^{R}(s)$ due to the sum of the low-mass vector meson spectral function $G_{V}(s)$. At higher energy $\left(s>s_{1}\right)$, besides the narrow charmonium and upsilon resonances, the spectral function is dominated by the contribution $\Pi^{C}(s)$ of the continuum quarkantiquark fluctuations. Then, the total spectral function $\Pi(s)$ can be written as

$$
\Pi(s)=\Pi^{R}(s)+\Pi^{C}(s)=\sum_{V=\rho, \omega, \phi, J / \psi, \psi^{\prime}} G_{V}(s)+\Pi^{C}(s) .
$$

Substituting Eq. (3) in Eq. (11), the $\sigma_{\gamma p}^{\text {had }}$ is written in terms of the resonance and the continuum contributions:

$$
\begin{aligned}
\sigma_{\gamma p}^{\text {had }}(k) & =\sigma_{\gamma p}^{R}(k)+\sigma_{\gamma p}^{C}(k)= \\
& =4 \pi \alpha_{e m} \sum_{V} \int_{s_{0}}^{s_{u}} \frac{d \mu^{2}}{\mu^{2}} G_{V}\left(\mu^{2}\right) \sigma_{V p}(k)+ \\
& +4 \pi \alpha_{e m} \int_{s_{1}}^{s_{u}} \frac{d \mu^{2}}{\mu^{2}} \Pi^{C}\left(\mu^{2}\right) \sigma_{q p}\left(\mu^{2}, k\right),
\end{aligned}
$$

where $\sigma_{V p}$ and $\sigma_{q p}$ are the interaction cross sections of the vector mesons and of the continuum quark-antiquark pairs respectively. In this work, $G_{V}(s)$ are derived directly from Eq. (2) by taking into account the experimental resonance widths [13]:

$$
G_{V}(s)=\frac{1}{\pi}\left(\frac{m_{V}}{g_{V}}\right)^{2} \frac{B_{V} m_{V} \Gamma_{V}(s)}{\left(s-m_{V}^{2}\right)^{2}+\left(m_{V} \Gamma_{V}(s)\right)^{2}},
$$

where $g_{V}$ are the $\gamma V$ coupling constants, $\Gamma_{V}(s)$ are the total hadronic widths of the resonances and $B_{V}$ are the branching ratios for the decay $V \rightarrow e^{+} e^{-}$[14]. The continuum contribution is written as:

$$
\Pi^{C}(s)=\frac{1}{12 \pi^{2}} \Sigma_{f} 3 q_{f}^{2}
$$

and the sum is extended over all quark flavors $f$ of fractional charge $q_{f}$ which are energetically accessible. In Fig. 3 the resonance and the continuum contributions to the spectral function are shown.

In order to evaluate the resonance contribution in Eq.( (4), experimental vector-meson proton cross sections $\sigma_{V p}(k)$ are considered. In particular, the $\sigma_{\rho p}(k)$ is derived from photoproduction data on hydrogen 115 . The $\rho$-meson photoproduction cross section is related to the elastic scattering of transversely polarized vector meson on nucleons by the VMD relationship and, through the optical theorem, to the total cross section $\sigma_{\rho p}$ :

$$
\left.\frac{d \sigma}{d t}(\gamma p \rightarrow \rho p)\right|_{t=0}=\frac{\alpha_{e m}}{64 \pi} \frac{4 \pi}{g_{\rho}{ }^{2}}\left(1+\eta_{\rho}{ }^{2}\right)\left(\frac{q_{\rho}}{q_{\gamma}}\right)^{2} \sigma_{\rho p}{ }^{2}
$$




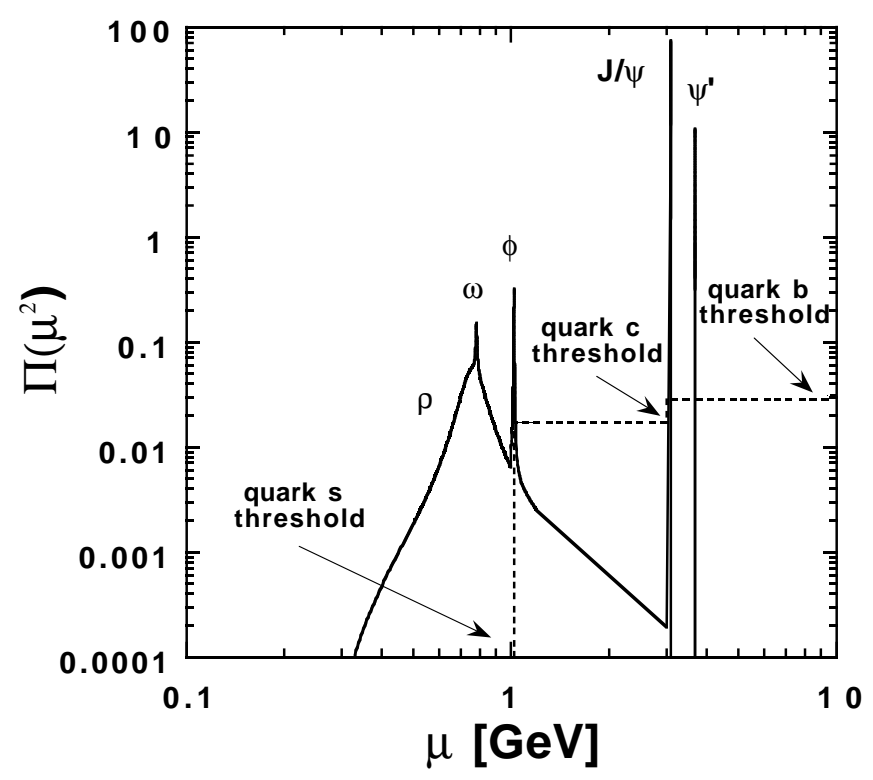

Figure 3: Continuum (dashed line) and resonance (solid line) contributions to the hadronic spectral function used in the model.

where $\eta_{\rho}$ is the ratio of the real to imaginary forwardscattering amplitude, and $q_{\rho}$ and $q_{\gamma}$ are the center of mass momenta of the $\rho p$ and $\gamma p$ systems at the same invariant collision energy $\sqrt{s}$ 16. The values of the $\eta_{\rho}$ and of the effective coupling constant $\frac{4 \pi}{g_{\rho}{ }^{2}}$ are from Ref. [17], where the effective $\rho$-coupling constant is reproduced by GVMD with physical coupling and the non diagonal $\rho p \rightarrow \rho^{\prime} p$ term. The $\sigma_{\rho p}$ is shown in Fig. 4 . As it is seen $\sigma_{\rho p}$ is higher at low energies; its energy behaviour is parameterized as

$$
\sigma_{\rho p}(k)=p_{1}+\frac{p_{2}}{\sqrt{k}},
$$

where $p_{1}=18 \mathrm{mb}$ and $p_{2}=27 \mathrm{mb} \mathrm{GeV}{ }^{1 / 2}$. The cross sections of the higher-mass vector mesons are fixed to $\sigma_{\omega p}(k)=\sigma_{\rho p}(k), \sigma_{\phi p}=12 \mathrm{mb}, \sigma_{J / \psi p}=2.2 \mathrm{mb}$ and $\sigma_{\psi^{\prime} p}=1.3 \mathrm{mb}$ [5].

The $\sigma_{q p}$ is determined by the transverse size of the $q \bar{q}$-fluctuations [5]:

$$
\begin{aligned}
\sigma_{q p}\left(\mu^{2}, k\right) & =\int_{0}^{1} \sigma_{q p}\left(\mu^{2}, k, \alpha\right) d \alpha= \\
& =\left(q_{1}+\frac{q_{2}}{\sqrt{k}}\right)\left[\frac{8}{\mu^{2}} \ln \left(\frac{1+x}{1-x}\right)+R_{c}^{2}(1-x)\right]
\end{aligned}
$$

where the integration is performed over the fraction $\alpha$ of the light-cone momentum carried by the quark 13 . Here $q_{1}$ and $q_{2}$ are free parameters, $x=\sqrt{1-\left(\frac{2}{\mu R_{c}}\right)^{2}}$ where $R_{c}$ is the maximum transverse size of the $q \bar{q}$-fluctuations. The continuum contribution $\sigma_{\gamma p}^{C}$ is derived by fitting to the Eq. (4) the proton photoabsorption cross section data 14 at photon energy higher than $5 \mathrm{GeV}$, where the direct contribution is assumed to be negligible.

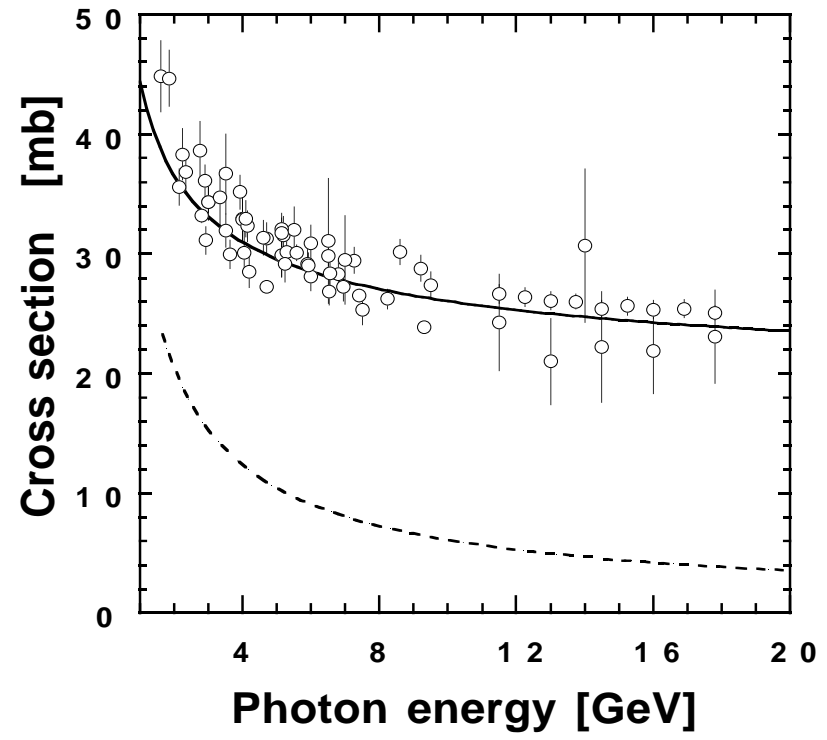

Figure 4: Fit (solid curve) to the $\rho$-meson interaction cross section for the proton $\sigma_{\rho p}$ derived from Refs. 15. (open circles). Dashed curve is the continuum interaction cross section $\sigma_{q p}$ derived from Eq.(9).

The direct contribution $\sigma_{\gamma p}^{d i r}$ is calculated as:

$$
\sigma_{\gamma p}^{d i r}=\sigma_{\gamma p}-\sigma_{\gamma p}^{h a d}
$$

where $\sigma_{\gamma p}(k)$ is parameterized as $\sigma_{\gamma p}=67.7 s^{0.08}+129 s^{-0.45}$ 18.

In Fig. 5 the result of the calculation for $\sigma_{\gamma p}$ in the energy range $1.65 \mathrm{GeV}<k<30 \mathrm{GeV}$ are presented together with the experimental data. The resonance $\sigma_{\gamma p}^{R}$ and the continuum $\sigma_{\gamma p}^{C}$ contributions to the total cross section $\sigma_{\gamma p}$ are also given. The $\rho$-meson accounts for about $85 \%$ of the resonance contribution, the $\omega$-meson for the $9 \%$, the $\phi$ meson for the $4 \%$. The small bump in the calculation that occurs at $k \sim 8 \mathrm{GeV}$ is due to the opening of charm channels which account for about $1 \%$. The $\sigma_{\gamma p}$ dir contribution to the total cross section is also shown in Fig. 5 .

The hadronic and the direct contributions to the total cross section for the neutron case have been also derived from the deuteron photoabsorption data 14 by using a procedure similar to the one described for the proton. This allows to evaluate the isospin weighted nucleon cross sections $\left(\sigma_{\gamma N}, \sigma_{\gamma N}^{h a d}, \sigma_{\gamma N}^{R}, \sigma_{\gamma N}^{C}, \sigma_{h N}, \sigma_{V N}\right.$ and $\left.\sigma_{q N}\right)$ for each nucleus.

\subsection{Photoabsorption on nuclei}

The nuclear photoabsorption cross section $\sigma_{\gamma A}$ is written as:

$$
\sigma_{\gamma A}(k)=\sigma_{\gamma A}^{d i r}(k)+\sigma_{\gamma A}^{h a d}(k) .
$$




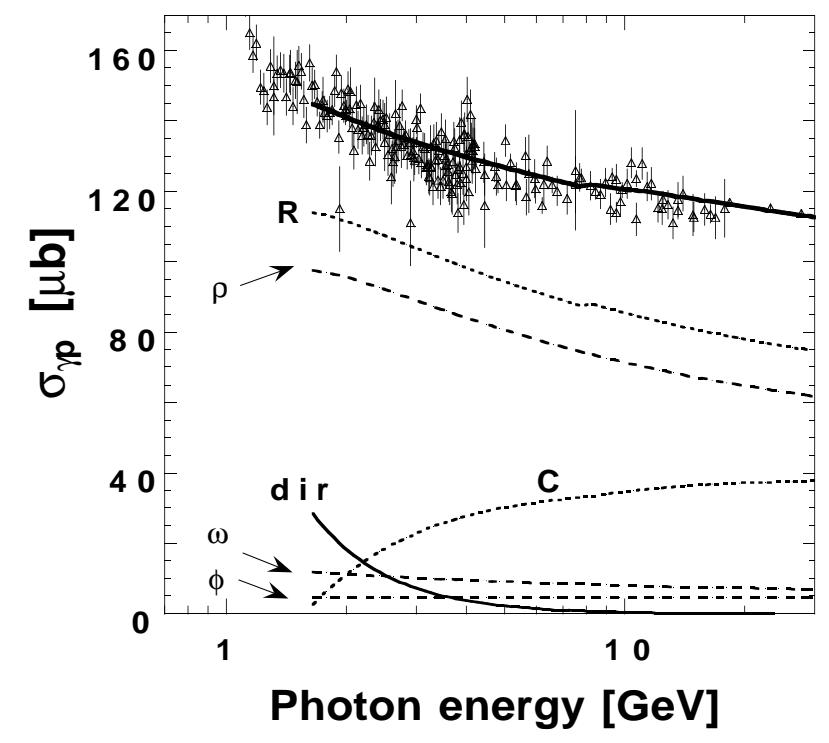

Figure 5: Predictions of the model (thick solid line) for the photoabsorption cross section on the proton. Dotted curves are the hadronic contributions due to resonance $(\mathrm{R})$ and to the continuum $(\mathrm{C})$. Dashed curves are the individual $\rho, \omega$ and $\phi$-mesons contributions. The contribution from direct processes (dir) is shown as a thin solid curve.

The direct term $\sigma_{\gamma A}^{d i r}(k)$ is equal to the incoherent sum of the corresponding terms on proton and neutron:

$$
\sigma_{\gamma A}^{d i r}(k)=Z \sigma_{\gamma p}^{d i r}(k)+N \sigma_{\gamma n}^{d i r}(k) .
$$

The hadronic term is derived by substituting in Eq. (1) the hadron-proton cross section $\sigma_{h p}$ with the hadron-nucleus cross section $\sigma_{h A}$ :

$$
\sigma_{\gamma A}^{h a d}(k)=4 \pi \alpha_{e m} \int_{s_{0}}^{s_{u}} \frac{d \mu^{2}}{\mu^{2}} \Pi\left(\mu^{2}\right) \sigma_{h A}\left(\mu^{2}, k\right) .
$$

Inside the nucleus the intermediate hadronic system undergoes a coherent scattering on bound nucleons. The interference between multiple scattering amplitudes reduces the hadron-nucleus cross section $\sigma_{h A}$ compared to $A \sigma_{h N}$ thus leading to shadowing. This process is described by the Glauber-Gribov multiple scattering formalism [19].

Considering the scattering on one up to five nucleons, $\sigma_{h A}$ is given by:

$$
\begin{aligned}
& \sigma_{h A}\left(\mu^{2}, k\right)=A \sigma_{h N}\left[1-a_{2}(A-1) \frac{\sigma_{h N}}{\pi \overline{r^{2}}} F_{2}(\epsilon)+\right. \\
& \quad+a_{3}(A-1)(A-2)\left[\frac{\sigma_{h N}}{\pi r^{2}}\right]^{2} F_{3}(\epsilon)- \\
& \quad-a_{4}(A-1)(A-2)(A-3)\left[\frac{\sigma_{h N}}{\pi \overline{r^{2}}}\right]^{3} F_{4}(\epsilon)+ \\
& \left.+a_{5}(A-1)(A-2)(A-3)(A-4)\left[\frac{\sigma_{h N}}{\pi \bar{r}^{2}}\right]^{4} F_{5}(\epsilon)\right]
\end{aligned}
$$

where $a_{n}$ are numerical coefficients which are strongly decreasing with $n, F_{n}(\epsilon)$ are functions of $\epsilon\left(\mu^{2}, k\right)=\sqrt{\overline{r^{2}}} / \lambda_{h}$ which depend on the nuclear density distribution. The quantity $\overline{r^{2}}$ is the rms electron-scattering radius given in Ref.20].

When $\lambda_{h} \ll \sqrt{\overline{r^{2}}}, F_{n}(\epsilon)$ approximately vanish and $\sigma_{h A}=$ $A \sigma_{h N}$. Otherwise there is shadowing and the shadowing cross section reduction $\Delta \sigma(k)=\sigma_{\gamma A}^{\text {had }}(k)-A \sigma_{\gamma N}^{\text {had }}(k)$ is given by

$$
\Delta \sigma(k)=\Delta \sigma^{C}(k)+\Delta \sigma^{R}(k)
$$

with

$$
\begin{aligned}
& \Delta \sigma^{C}(k)=4 \pi \alpha_{e m} A(A-1) \times \\
& \times\left[\frac{a_{2}}{\pi \bar{r}^{2}} \int_{s_{1}}^{s_{u}} \frac{d \mu^{2}}{\mu^{2}} \Pi^{C}\left(\mu^{2}\right) \Sigma_{q N}^{(2)}\left(\mu^{2}, k\right) F_{2}(\epsilon)-\right. \\
& a_{3} \frac{(A-2)}{\left[\pi \overline{r^{2}}\right]^{2}} \int_{s_{1}}^{s_{u}} \frac{d \mu^{2}}{\mu^{2}} \Pi^{C}\left(\mu^{2}\right) \Sigma_{q N}^{(3)}\left(\mu^{2}, k\right) F_{3}(\epsilon)+ \\
& a_{4} \frac{(A-2)(A-3)}{\left[\pi \bar{r}^{2}\right]^{3}} \int_{s_{1}}^{s_{u}} \frac{d \mu^{2}}{\mu^{2}} \Pi^{C}\left(\mu^{2}\right) \Sigma_{q N}^{(4)}\left(\mu^{2}, k\right) F_{4}(\epsilon)- \\
& \left.a_{5} \frac{(A-2)(A-3)(A-4)}{\left[\pi r^{2}\right]^{4}} \int_{s_{1}}^{s_{u}} \frac{d \mu^{2}}{\mu^{2}} \Pi^{C}\left(\mu^{2}\right) \Sigma_{q N}^{(5)}\left(\mu^{2}, k\right) F_{5}(\epsilon)\right]
\end{aligned}
$$

and

$$
\begin{aligned}
& \Delta \sigma^{R}(k)=4 \pi \alpha_{e m} A(A-1) \times \\
& \times \sum_{V}\left[\frac{a_{2}}{\pi \overline{r^{2}}} \int_{s_{0}}^{s_{u}} \frac{d \mu^{2}}{\mu^{2}} G_{V}\left(\mu^{2}\right) \Sigma_{V N}^{(2)}(k) F_{2}(\epsilon)-\right. \\
& a_{3} \frac{(A-2)}{\left[\pi \overline{r^{2}}\right]^{2}} \int_{s_{0}}^{s_{u}} \frac{d \mu^{2}}{\mu^{2}} G_{V}\left(\mu^{2}\right) \Sigma_{V N}^{(3)}(k) F_{3}(\epsilon)+ \\
& a_{4} \frac{(A-2)(A-3)}{\left[\pi \overline{r^{2}}\right]^{3}} \int_{s_{0}}^{s_{u}} \frac{d \mu^{2}}{\mu^{2}} G_{V}\left(\mu^{2}\right) \Sigma_{V N}^{(4)}(k) F_{4}(\epsilon)- \\
& \left.a_{5} \frac{(A-2)(A-3)(A-4)}{\left[\pi \overline{r^{2}}\right]^{4}} \int_{s_{0}}^{s_{u}} \frac{d \mu^{2}}{\mu^{2}} G_{V}\left(\mu^{2}\right) \Sigma_{V N}^{(5)}(k) F_{5}(\epsilon)\right]
\end{aligned}
$$

where

$$
\Sigma_{q N}^{(i)}\left(\mu^{2}, k\right)=\int_{0}^{1} d \alpha\left[\sigma_{q N}\left(\mu^{2}, \alpha, k\right)\right]^{i},
$$

and for each nucleus

$$
\begin{aligned}
& {\left[\sigma_{q N}\left(\mu^{2}, \alpha, k\right)\right]^{i}=\left[\frac{Z \sigma_{q p}\left(\mu^{2}, \alpha, k\right)+N \sigma_{q n}\left(\mu^{2}, \alpha, k\right)}{A}\right]^{i}} \\
& \Sigma_{V N}^{(i)}(k)=\left[\sigma_{V N}(k)\right]^{i}=\left[\frac{Z \sigma_{V p}(k)+N \sigma_{V n}(k)}{A}\right]^{i}
\end{aligned}
$$

Two different parameterizations of the nuclear density are used in the evaluation of the functions $F_{n}$, specifically a Gaussian and a uniform density distributions for light and heavy nuclei, respectively. In both cases the experimental average nuclear density and $\overline{r^{2}}$ values are well 
reproduced 13. Being each term in Eq. (14) proportional to $A^{\frac{n+2}{3}}$, the third, fourth, and fifth terms give a non negligible contribution only for the heavy nuclei. Then for the light nuclei the first and second terms in Eq. (14) are only considered.

The results of the calculation are shown in Figs. 6 and 7 for five nuclei. The comparison with the data is performed for both the photonuclear cross section and the ratio between photonuclear and photonucleon cross sections. As it is seen the results are in slightly better agreement with the data with respect to previous models [5, 6, 7]. However the calculation still shows a stronger energy dependence than data. In particular, at low energy it overestimates the experimental result thus suggesting the need of further mechanisms for the description of the process.

\section{Medium effects on the hadronic spectral function}

The calculation described in the previous section is based on the assumption that the spectral function of the hadronic fluctuation of the photon does not change inside the nuclear medium. In order to improve the phenomenological description of the low energy photonuclear data, the effect of the possible hadronic mass modification in the nuclear medium is now considered.

The $\rho$-meson mass modification in the nuclear medium is predicted by several theoretical approaches which consider effective chiral Lagrangians, in-medium scaling properties based on QCD sum rules, quark bag models combined with quantum hadrodynamics (for a recent review see Ref. 21]). Many of these theories predict a mass reduction $\delta m_{\rho}$ proportional to the average nuclear density and amounting up to about 100-200 MeV for the nuclear matter density. The decrease of the $\rho$-meson mass inside the nucleus increases the coherence length $\lambda_{\rho}=2 \mathrm{k} / \mathrm{m}_{\rho}^{2}$ and thus decreases the energy threshold for the shadowing. Other theories predict a broadening or a complete distortion of the in-medium $\rho$-meson mass distribution.

Considering a possible $\rho$-meson mass shift in nuclei, a fit to the photonuclear absorption data is performed by using the previously described calculation. In the spectral function $\Pi\left(\mu^{2}\right)$ of Eq. (13), the $\rho$-meson mass $m_{\rho}$ is replaced by $m_{\rho}+\delta m_{\rho}$, with $\delta m_{\rho}$ free parameter. In order to reduce the number of free parameters in the fitting procedure, no mass modifications of other vector-mesons are considered since their contributions are small. The fits are shown in Figs. 6 and 7 ; the relevant $\chi^{2}$ improves by about a factor of two with respect to the calculations with $\delta m_{\rho}=0$. It is worth to mention that also a distortion of the $\rho$-meson mass distribution, which enhances the low mass hadronic spectral function, will result in a better agreement with the experimental data.

The values of the $\delta m_{\rho}$ obtained from the fits are given in Table 1: they range from $-63 \mathrm{MeV}$ to $-163 \mathrm{MeV}$ and the shift in carbon is more than a factor of two bigger than in lead. The values of the $\delta m_{\rho}$ obtained for the lightest nuclei are significantly larger than most of the theoretical expectations, while are in qualitative agreement with a recent measurement performed via the ${ }^{3} \mathrm{He}\left(\gamma, \pi^{+} \pi^{-}\right) X$ reaction 22, 23] which suggests a $\sim 160 \mathrm{MeV}$ reduction of the $\rho$-mass in ${ }^{3} \mathrm{He}$. This reduction is so large that cannot be explained by the mean field picture of nuclear matter [24]. In this latter reference, unlike all other calculations which consider infinite nuclear matter, the experimental charge density distributions are used, resulting in a shift in ${ }^{4} \mathrm{He}$ about a factor of two bigger than in ${ }^{12} \mathrm{C}$ due to the higher ${ }^{4} \mathrm{He}$ core density. Also a recent calculation that accounts for the local density distributions in ${ }^{3} \mathrm{He}$, shows a substantial changes in the $\rho$-meson mass [25]. In this respect the large shift observed in the fit of the light nuclei photoabsorption data could be ascribed to the high core density, while for the heavier nuclei the mass-shifts agree with the theoretical predictions which account for the mean nuclear field alone.

Moreover, the higher local density distributions for the lighter nuclei can reduce the local intranucleon distance $d_{N N}$, thus accounting for an earlier onset of the shadowing effect on these nuclei.

Table 1: $\rho$-meson mass shifts $\delta m_{\rho}$ extracted from the photoabsorption data fits. The errors indicate the statistical and the systematic uncertainties.

\begin{tabular}{lclc}
\hline \hline Nucleus & $\delta m_{\rho}[\mathrm{MeV}]$ & Nucleus & $\delta m_{\rho}[\mathrm{MeV}]$ \\
\hline $\mathrm{C}$ & $-163 \pm 14 \pm 50$ & $\mathrm{Sn}$ & $-115 \pm 17 \pm 53$ \\
$\mathrm{Al}$ & $-133 \pm 11 \pm 40$ & $\mathrm{~Pb}$ & $-63 \pm 20 \pm 62$ \\
$\mathrm{Cu}$ & $-104 \pm 14 \pm 57$ & & \\
\hline \hline
\end{tabular}

\section{Conclusions}

Total photoabsorption cross sections for nucleon and nuclei are calculated in the energy range $1.65-30 \mathrm{GeV}$. The process is described taking into account both the direct and the hadronic fluctuation interactions of the photon. The latter is computed with a hadronic spectral function which includes the effective $\rho$-coupling constant, the finite width of vector-meson resonances and the quark-antiquark continuum. Realistic and energy dependent interaction cross section for the $\rho$-meson is derived from photoproduction data. The shadowing effect is evaluated in the framework of a Glauber-Gribov multiple scattering theory up to the $5^{\text {th }}$ order.

The low energy onset of the shadowing effect is interpreted as a possible signature of a modification of the hadronic spectral function in the nuclear medium. In particular, a decrease of the $\rho$-meson mass in nuclei is suggested for a better description of the experimental data. 
This reduction is larger for the light nuclei and cannot be accounted for by mean field consideration alone.

\section{Acknowledgments}

We would like to express our gratitude to K. Saito and A. Sibirtsev for useful discussions, and to A. Bhattacharyya for providing us with results prior of publication.

\section{References}

[1] S. J. Brodsky and J. Pumplin, Phys. Rev. 182, 1794 (1969); K. Gottfried and D. R. Yennie, Phys. Rev. 182, 1595 (1969).

[2] D. O. Caldwell et al., Phys. Rev. Lett. 23, 1256 (1969); V. Heynen, H. Meyer, B. Naroska and D. Notz, Phys. Lett. B 34, 651 (1971); G. R. Brookes et al., Phys. Rev. D 8, 2826 (1973); D. O. Caldwell et al., Phys. Rev. D 7, 1362 (1973); S. Michalowski et al., Phys. Rev. Lett. 39, 737 (1977); E. A. Arakelyan et al., Phys. Lett. B 79, 143 (1978); T. H. Bauer, R. D. Spital, D. R. Yennie and F. M. Pipkin, Rev. Mod. Phys. 50, 261 (1978); D. O. Caldwell et al., Phys. Rev. Lett. 42, 553 (1979).

[3] P. Distas and G. Shaw, Nucl. Phys. B 113, 246 (1976); G. Shaw, Phys. Rev. D 47, R3676 (1993).

[4] N. Bianchi et al., Phys. Rev. C 54, 1688 (1996); M. Mirazita et al., Phys. Lett. B 407, 225 (1997); V. Muccifora et al., Phys. Rev. C submitted and nucl$\mathrm{ex} / 9810015$.

[5] G. Piller, W. Ratzka and W. Weise, Z.Phys. A 352, 427 (1995).

[6] S. Boffi, Ye. Golubeva, L. A. Kondratyuk and M. I. Krivoruchenko, Nucl. Phys. A 606, 421 (1996); S. Boffi, Ye. S. Golubeva, L. A. Kondratyuk, M. I. Krivoruchenko and E. Perazzi, Phys. of At. Nucl. 60, 1193 (1997).

[7] R. Engel, J. Ranft and S. Roesler Phys. Rev. D 55, 6957 (1997).

[8] G. Piller and W. Weise, Phys. Rev. C 42, 1834 (1990).

[9] G. E. Brown and M. Rho, Phys. Rev. Lett. 66, 2720 (1991).

[10] T. Hatsuda and S. H. Lee, Phys. Rev. C 46, R34 (1992).

[11] R. Rapp, G. Chanfray and J. Wambach, Nucl. Phys. A617, 472 (1997).

[12] F. Klingl, N. Kaiser and W. Weise, Nucl. Phys. A624, 527 (1997).
[13] M. Mirazita, Ph.D. Thesis, Frascati IR LNF-98/006.

[14] Particle Data Group (R.M. Barnett et al.), Phys. Rev. D 54, 191 (1996).

[15] ABBHHM Collaboration , Phys. Rev. 175, 5, 1669 (1968); R. Erbe et al., Phys. Lett. B 27, 54 (1968); W. J. Jones et al., Phys. Rev. Lett. 21586 (1968); H. Blrchschmidt et al., Il Nuovo Cimento A 4, 1349 (1968); M. Davier et al. , Phys. Rev. Lett. 21, 841 (1968); G. McClellan et al., Phys. Rev. Lett. 22, 374 (1969); H. Alvensleben et al., Phys. Rev. Lett. 23, 1058 (1969); R. Anderson et al., Phys. Rev. D 1, 27 (1970); G. McClellan et al., Phys. Rev. D 4, 2683 (1971); Y. Eisenberg et al., Phys. Rev. D 5, 15 (1972); W. Struczinski et al., Nucl. Phys. B 47, 437 (1972); J. Ballam et al., Phys. Rev. D 7, 3150 (1973); W. Struczinski et al., Nucl. Phys. B 108, 45 (1976); D. Aston et al., Nucl. Phys. B 209, 56 (1992).

[16] L. A. Kondratyuk, A. Sibirtsev, W. Cassing, Ye. S. Golubeva and M. Effenberger, Phys. Rev. C 58, 1079 (1998).

[17] A. Pautz and G. Shaw, Phys. Rev. C 57, 5 27, 2648 (1998).

[18] A. Donnachie and P. V. Landshoff, Phys. Lett. B 296, 227 (1992).

[19] V. N. Gribov, JETP 30, 709 (1970).

[20] C.W. De Jager, H. De Vries, and C. De Vries, At. Data and Nucl. Data Tables 14, 479 (1974).

[21] W. Cassing and E. L. Bratkovskaya, Phys. Rep. 308, 65 (1999) and references therein.

[22] G. J. Lolos et al., Phys. Rev. Lett. 80, 241 (1998).

[23] G. M. Huber, G. J. Lolos and Z. Papandreou, Phys. Rev. Lett. 80, 5285 (1998).

[24] K. Saito, K. Tsushima and A. W. Thomas, Phys. Rev. C 56, 566 (1997); K. Saito, K. Tsushima and A. W. Thomas, Phys. Rev. C 55, 2637 (1997).

[25] A. Bhattacharyya, S. K. Ghosh and S. Raha, nuclth/9902060. 


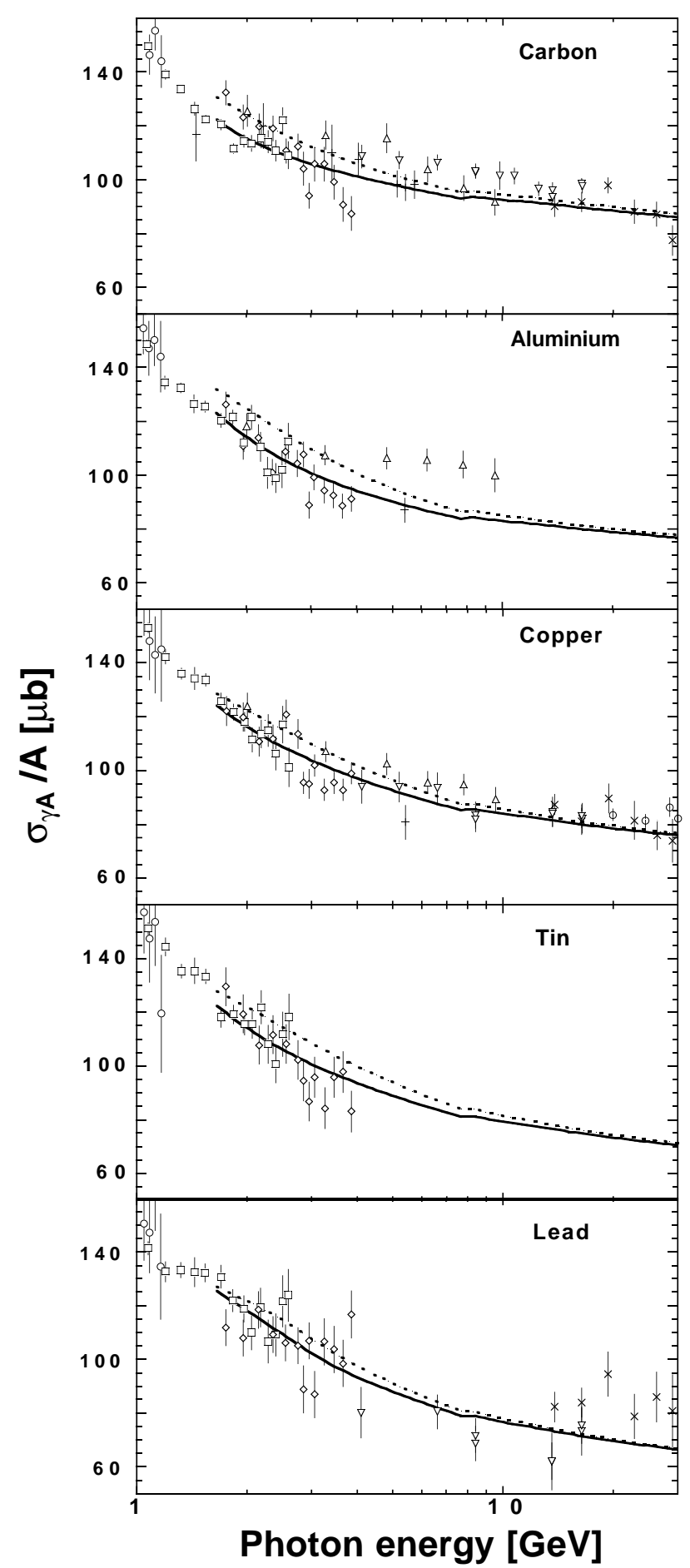

Figure 6: Results of the calculation for $\sigma_{\gamma A} / \mathrm{A}$ (dotted curves). The solid curves are the result of the fit obtained considering the $\rho$-meson mass shift. Different symbols refer to different experiments.

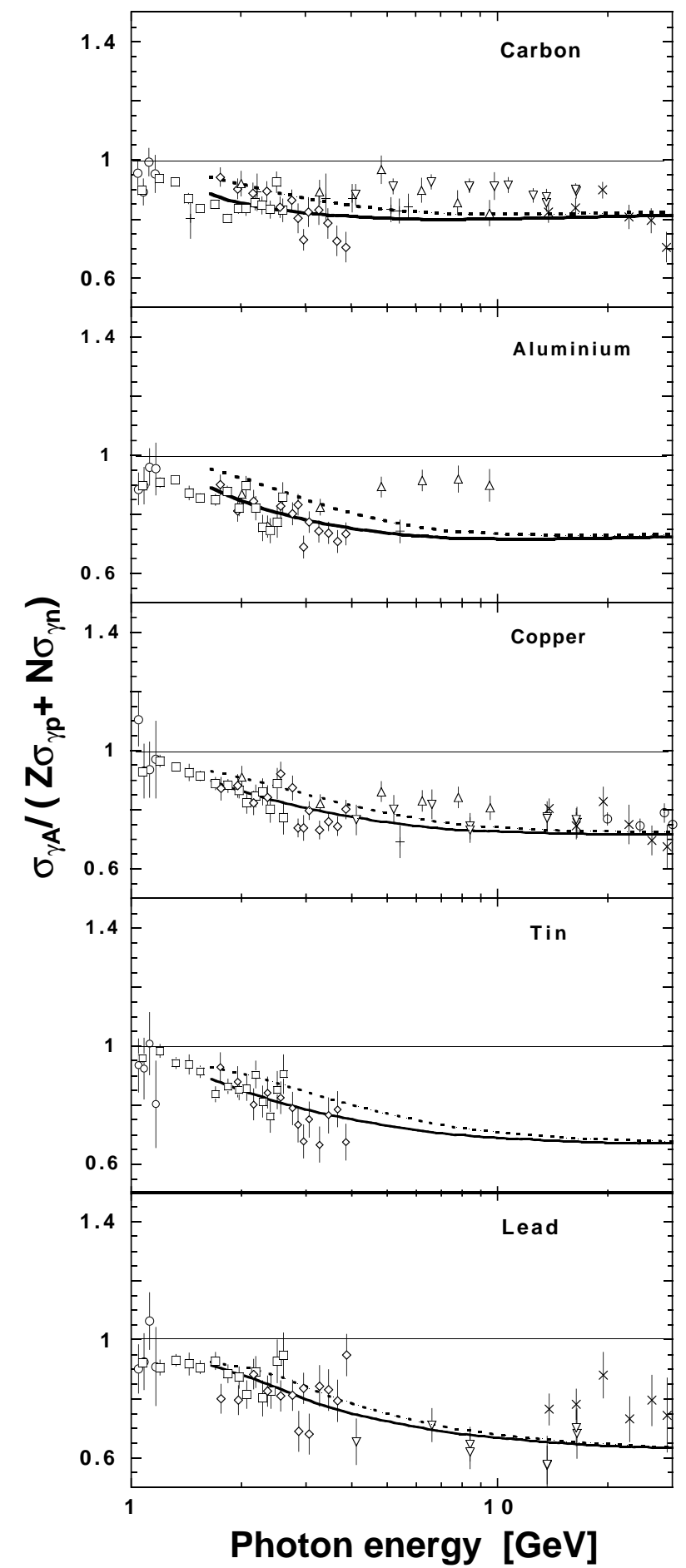

Figure 7: Shadowing effect $\sigma_{\gamma A} /\left(Z \sigma_{\gamma p}+N \sigma_{\gamma n}\right)$. Same notations as in Fig. 6. 